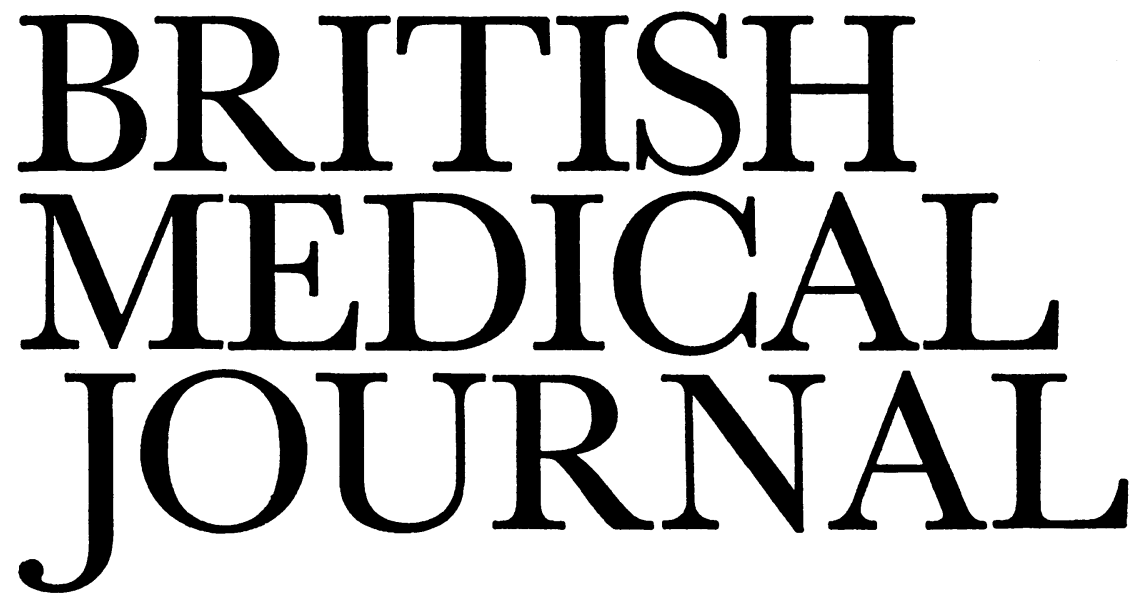

LONDON SATURDAY 7 OCTOBER 1972

\title{
Diagnosis of Gout
}

Gout may be defined as the articular manifestation of hyperuricaemia. It is due to a crystal-induced synovitis. ${ }^{1-3}$ While untreated true gout is almost invariably associated with hyperuricaemia, a patient may have hyperuricaemia without suffering gout.

Serum levels of uric acid in the general population follow a unimodal distribution curve with a mean of approximately $4-4.5 \mathrm{mg} / 100 \mathrm{ml} .45 \mathrm{Up}$ to $9 \%$ of males and $3.5 \%$ of females have serum levels of uric acid above the arbitrary upper limit of normal of $6.5 \mathrm{mg} / 100 \mathrm{ml}$. But true gout occurs in only about $0.5 \%$ of Europeans. 467

Urate is excreted through the kidneys, initially by glomerular filtration with subsequent reabsorption in the proximal tubule and finally by distal tubular secretion. This may be impaired by commonly used drugs such as thiazide diuretics and small doses of aspirin, ${ }^{89}$ leading to hyperuricaemia. The serum uric acid level is often measured as a routine in the investigation of any joint discomfort. Thus gout may be erroneously diagnosed in patients with minor rheumatic complaints and incidental, possibly drug-induced, hyperuricaemia.

The diagnosis of true gout is initially clinical. The history of acute attacks of exquisitely severe pain in a joint, accompanied by swelling, redness, and overlying dry skin and gross tenderness, is typical. Untreated these attacks resolve spontaneously in five to seven days, leaving a normal joint. Classically the first metatarsal-phalangeal joint is affected ("podagra"), but identical attacks may occur in the other small joints of the hands and feet, wrists, ankles, knees, or elbows. Presenting attacks do not occur in the shoulders or hips. Often the attack has subsided by the time medical advice is sought, and then only the history and sometimes dry desquamating skin over the affected joint remain, with a subsequent discovery of hyperuricaemia It is rare that a careful history, with the finding of hyperuricaemia, fails to lead to the correct diagnosis, but in difficult cases the joint fluid should be examined. The presence of negatively birefringent needle-shaped crystals of sodium biurate in the fluid when examined by polarized light microscopy ${ }^{13}$ confirms the diagnosis.

For population studies the following criteria for the diagnosis of gout were proposed in $1966^{10}$ : either (1) the presence of uric acid crystals in synovial fluid or of urate deposition in the tissues; or (2) two or more of the fol- lowing: (a) at least two typical attacks, (b) podagra, (c) clinical tophus formation, $(d)$ response to colchicine.

A clinical response to colchicine in full dosage has been proposed as a therapeutic trial to confirm the diagnosis, ${ }^{11}$ but in routine clinical practice it is found that either phenylbutazone $\mathrm{e}^{12}$ or indomethacin are effective in the treatment of the acute attack. Radiographic changes are not a sensitive criterion of diagnosis for gout. ${ }^{10}$ The "punched-out cystic lesions" described as the characteristic radiographic feature of gout represent juxta-articular deposits of solid urate within bone. The diagnosis should have been made long before these radiographic changes develop. Similarly, subcutaneous tophi are usually a late feature and may often be prevented or reduced by early diagnosis and treatment aimed at lowering the levels of uric acid in the serum. Though affected joints return to normal between acute attacks in the early stages of the disease, repeated deposition of urate eventually damages the cartilage, produces permanent changes of (secondary) degenerative joint disease, and sometimes leads to bony fusion. ${ }^{13}$

Gout can nearly always be controlled by either uricosuric drugs or allopurinol, which lower the serum uric acid. It is essential, therefore, to make the diagnosis and not to treat all patients with hyperuricaemia who happen to have trivial, non-gouty, rheumatic complaints under the erroneous diagnosis of gout. Similarly it is not sufficient merely to treat acute attacks symptomatically as they occur. 1415

Problems of diagnostic failure in gouty arthritis have recently been discussed by I. Zimmerman-Gorska and A. Koscianska. ${ }^{16}$ They reviewed the histories of 34 patients (32 with definite and 2 with possible gout), of whom only 9 were correctly diagnosed initially. Most of the remainder had originally been diagnosed as having rheumatic fever, rheumatoid arthritis, or osteoarthrosis. Yet 28 of the 34 had a history of "typical joint manifestation." 32 had podagra, 10 had tophi, and 32 were hyperuricaemic. Twentytwo were treated with colchicine, of whom 19 responded. This paper shows, as mentioned above, that the diagnosis is largely clinical, confirmed by finding hyperuricaemia.

The principal differential diagnosis is pyrophosphate arthropathy ("pseudogout"). This also is a crystal-deposition disease, the crystals being calcium pyrophosphate dihydrate. The patient may present with acute attacks of joint pain.17 Podagra is unusual; hips and shoulders may be involved; and 
$x$-ray examination will show typical chondrocalcinosis articularis.

1 Faires, J. S., and McCarty, D. J. Jr., Clinical Research, 1961, 9, 329. McCarty, D. J. Jr., and Hollander, J. L., Annals of Internal Medicine, $1961,54,452$

Seegmiller, J. E., Howell, R. R., and Malawista, S. E., Fournal of the American Medical Association, 1962, 180, 469.

- Popert, A. J., and Hewitt, J. V., Annals of Rheumatic Diseases, 1962, 21,

Hall, A. P., Barry, P. E., Dawber, T

Fournal of Medicine, 1967, 42, 27. Diseases, 1960, 19, 120.
.

7 Kellgren, J. H., Annals of Rheumatic Diseases, 1964, 23, 109.
K Klemperer, F., and Bauer, W., Fournal of Clinical Investigation, 1944, 23, 950 . - Yu, T-F, and Gutman, A. B., Fournal of Clinical Investigation, 1959, 38,

10 Bennett, P. H., and Wood, P. H. N., eds. Population Studies of the Rheumatic Diseases, p. 457. Proceedings of the 3rd international symposium, New York, 1966. Amsterdam, Excerpta Medical Foundation, 1968.

11 Wallace, S. L., Bernstein, D., and Diamond, H., Fournal of the American Medical Association, 1967, 199, 525.

12 Gutman, A. B., Arthritis and Rheumatism, 1965, 8, 911.

13 Hughes, G. R., Barnes, C. G., and Mason, R. M., Annals of Rheumatic Diseases, 1968, 27, 67 .

14 Scott, J. T., British Medical fournal, 1969, 3, 456.

15 Barnes, C. G., Practitioner, 1972, 208, 101.

16 Zimmerman-Gorska, I., and Koscianska, J., Annals of Clinical Research,

17 Currey, H. 95. L. F., Key, J. J., Mason, R. M., and Swettenham, K. V., Annals of Rheumatic Diseases, 1966, 25, 295.

\section{Respiratory Distress Syndrome}

Respiratory distress syndrome remains a serious disorder of newborn babies. ${ }^{12}$ About $10-15 \%$ of infants with a birth weight of $2,500 \mathrm{~g}$ or less develop it. ${ }^{3}$ Though birth by caesarean section, particularly after vaginal bleeding, and being born of a diabetic mother increase the likelihood of the syndrome, prematurity is by far the commonest condition associated with it. There is no specific treatment, and between 20 and $40 \%$ of infants with the diagnosis succumb. Even in infants of 35 weeks gestation or more the mortality is $10 \% .4$ For the obstetrician considering the timing of elective and possibly premature delivery, precise indication of the risk of the infant's developing this syndrome would clearly be of value.

The aetiology of the disorder remains obscure, but it seems to be linked with some still unrecognized prenatal disturbance or impaired development in systems for the production of fibrinolytic activators, for the control of pulmonary blood flow and capillary permeability, or for surfactant synthesis. ${ }^{2}$ E. O. R. Reynolds and colleagues ${ }^{5}$ concluded that the crucial factor in causing respiratory distress is deficiency of surfactant, a lipoprotein which reduces surface tension within the lung. This lipoprotein facilitates expansion during inspiration and prevents pulmonary collapse during expiration. Surfactant is formed in alveolar cells, ${ }^{6}$ and its main surface-active component is dipalmitoyl lecithin. ${ }^{7}$ Its increased accumulation in the alveoli towards term is mirrored in a sharply rising concentration in the amniotic fluid from the 33rd week of pregnancy. The increase in concentration is not paralleled by an increase in concentration of another surface-active phospholipid sphingomyelin, and thus it was suggested that the relative concentration of these phospholipids in amniotic fluid could provide an index of the production of pulmonary surfactant and of the likelihood of the syndrome appearing if delivery is not postponed. ${ }^{8}$
The validity of this hypothesis and its usefulness in clinical practice were supported by the work of $\mathbf{C}$. R. Whitfield and his colleagues. 9 They found the lecithin/sphingomyelin ratio to be $0.8-2.0$ up to 32 weeks gestation, with a wide range thereafter, but at least 1.5 at 38 weeks and 2.0-9.0 at term. When the ratio was determined within 48 hours of delivery it was greater than 2.0 in 102 infants who did not subsequently develop the syndrome, less than 1.5 in 10 of whom 8 did develop it, and between 1.5 and 2.0 in 16 of whom 4 were affected. They added the notable observation that in pregnancies complicated by diabetes mellitus and severe rhesus isoimmunization the expected terminal rise in the ratio did not occur, and indeed in 3 out of 10 pregnancies of diabetic women a fall in the ratio was observed.

S. G. Bhagwanani and co-workers ${ }^{10}$ found chromatographic assessment of the lecithin/sphingomyelin ratio to be difficult but that quantitation of amniotic lecithin correlated with subsequent development of the syndrome. Ninety-seven infants, including 12 of low birth weight in whom samples of amniotic fluid obtained within 24 hours of delivery had contained more than $3.5 \mathrm{mg}$ of lecithin/100 $\mathrm{ml}$, did not develop respiratory distress syndrome, while 12 infants with levels of $0 \cdot 6-3.4 \mathrm{mg} / 100 \mathrm{ml}$ and one infant with Pierre Robin syndrome and a level of $6.9 \mathrm{mg} / 100 \mathrm{ml}$ were affected.

Both of these methods require skilled technical assistance and complex and expensive equipment, and they are timeconsuming. J. A. Clements and colleagues ${ }^{11}$ now describe in detail a simple, inexpensive, rapid, semiquantitative test for surfactant in which the ability of the amniotic fluid to form a stable foam is determined in $47.5 \%$ ethanol. False positive results are obtained if the amniotic fluid is contaminated with meconium, serum of more than $7 \%$ final concentration, ascitic fluid, vaginal fluid, or dirty glassware. In a double-blind field trial 68 infants whose amniotic fluid tested 24 hours before birth gave a positive test at $1 / 2$ dilution were not affected by respiratory distress syndrome, but all whose test at $1 / 1$ dilution was negative were affected. Of 13 infants whose tests were positive at $1 / 1$ dilution and negative at $1 / 2$ eight developed the syndrome. The results correlated well with the lecithin/sphingomyelin ratio, ${ }^{8}$ and the authors concluded that the test is sufficiently reliable and informative to merit extensive clinical trial. Failure of complete correlation between the results of these tests done within 24-48 hours of birth and the baby's respiratory state may be due to the short turnover time of surfactant. ${ }^{12}$

Though amniocentesis is not without risk, further evaluation of these tests, with other estimates of fetal maturity, ${ }^{13}$ seems to be indicated when the timing of elective interruption of pregnancy is considered in such conditions as chronic placental insufficiency or assumed postmaturity when the exact duration of gestation is unknown. In the management of pregnancy in cases of maternal diabetes serial determinations of amniotic surfactant may prove helpful, for though infants dying of respiratory distress syndrome associated with maternal diabetes appear comparatively well endowed with surfactant at the time of death ${ }^{14}$ they may have been deficient earlier. The test would seem to be most useful at present in cases of severe rhesus isoimmunization, because intrauterine transfusion is a feasible alternative to delivery at 33-35 weeks, at which time the infant is at risk of developing respiratory distress syndrome.

The development of better methods for the effective inhibition of uncomplicated premature labour ${ }^{15}$ would extend the value of the test. But further study of the surfactant system and of how to influence it pharmacologically is 